\title{
Supportive robotics impacting workplace practice
}

\section{Emergency department nursing}

\author{
Adam Morse, Ryan Chan
}

The development of drones, artificial intelligence, and robotics has resulted in a rise in intelligent supportive robotics that can be integrated into a variety of professions, including healthcare. Although the healthcare industry has evolved to incorporate intelligent elements such as electronic medical records into all healthcare systems in North America, little emphasis has been placed on the integration of supportive robotics. The premise of this commentary is to explore how the integration of supportive robotics may influence current workplace practices in emergency department (ED) nursing.

The evolution of assistive technology is expanding beyond the traditional aim of supporting those with disability and towards becoming an extension of our conceptual abilities. ${ }^{1}$ One form of assistive technology is supportive robotics, which is the use of robots to support individuals, such as nurses, in completing required tasks more efficiently. Merriam-Webster defines a robot as an independent operation a machine resembling a living creature capable of performing complex actions. ${ }^{2}$ The use of supportive robotics results in a decrease in the level of time and effort committed to completing tasks, and nurses are able to redirect their time to engage and focus on providing more direct patient care. For instance, self-driving patient beds, autonomous function stocking robots, and artificial nursing humanoids are examples of supportive robotics that can be utilized by health care organizations and departments to support the workload and responsibilities of nursing staff. Research has begun to examine the influence of certain technologies on decision making in nursing, including access to medical records, ${ }^{3,4}$ the use of telecommunication devices, ${ }^{5}$ suggestive nursing diagnoses using predictive models generated through data mining, ${ }^{6}$ and a proposed triage system that could theoretically utilize humanoid artificial agents. ${ }^{7}$ This literature does not examine nursing decision-making processes when utilizing supportive robotics in ED settings, or even the measurable benefits of introducing such robotics into ED settings.

The Canadian Institute for Health Information (CIHI) has indicated that between 2016 and 2017 there were 13.98 million ED visits, with $10 \%$ lasting longer than 7.8 hours. ${ }^{8}$ This is an $11 \%$ increase in length of stay in the ED for admitted patients compared to the previous year. ${ }^{8}$ When examining the past seven years, there has been a $17.5 \%$ increase in the number of patients admitted from the ED, with a $44.1 \%$ increase in high-acuity discharge patient visits. ${ }^{9}$ This reveals a highly demanding and high-stress work environment when compared to five years ago, when there were only 11.6 million visits to the $\mathrm{ED} .{ }^{8} \mathrm{ED}$ staffing has remained unchanged regardless of population growth, employing $10.7 \%$ of nurses with no noted increase in employment despite a 1.02 million visit increase in 5 years and a 2.37 million visit increase in the last decade. ${ }^{8,9}$ With ED visits increasing at double the rate of population growth, an opportunity is presented for technology to alleviate nurses' workload and combat the increased demands on healthcare professionals. ${ }^{10}$ Additionally, the elevated staffing requirements resulted from sick time and stress leave may substantially benefit from the introduction of various supportive robotics that possess unique capabilities and functionalities.

For instance, automated pharmacy robots possess the capability to autonomously administer medication quickly, accurately, and safely, a task regularly performed by a registered nurse. ${ }^{11}$ Meanwhile, social robots can socially interact with individuals through the building and integration of various social cues. Social robots are most commonly used to assist individuals with varying degrees of dementia. As this form of robotics has the ability to engage in social interactions and communicate with individuals, it provides an opportunity for nurses to spend their time performing clinical and therapeutic tasks that require a higher level of skill and expertise.

In addition to providing nurses with opportunities to distribute their time more effectively in a high-stress and demanding work environment, supportive robotics may also play a key role in the re-distribution of resources, most notably financial resources associated with staffing requirements (eg sick time and stress leave). Given the demanding nature and acuity of the EDs, staffing and human resources have always been a major source of concern for departmental leaders. As a result, robotics may serve as a potential option to address staffing levels and yield greater financial sustainability.

Despite the benefits associated with the implementation of supportive technologies, it does not come without risk. The current use and adoption of supportive robotics has raised concerns regarding the level of accuracy, precision, and safety in its operational capabilities; in extreme cases, this has unfortunately resulted in tragic events (eg security bot ran over toddler, ${ }^{12}$ Tesla driver killed when warnings ignored ${ }^{13}$ ). Further, the lack of current knowledge examining the influence of supportive technologies on the nursing decision-making process must be addressed. Many nurses work in acute care settings that require a high level of critical thinking and thought processing to complete tasks in an ever-changing dynamic environment. ${ }^{14}$

Further research should examine why humans fail to intervene at various points of interaction with robotics to mitigate concerns that have been identified in the private sector. This may be due to the development of comfort or habitual patterns resulting in a high level of complacency, creating an error and possible injury during task completion. ${ }^{15}$ This is a concept that should be extrapolated to 
the integration of robotics into nursing. Developing a high level of complacency is extremely concerning given the level of intensity and associated risk within the ED. It is for these reasons that further research examining the influence of supportive robotics on human decision-making is required.

Regardless of the noted lack of research, there still exists the beginnings of a crisis within EDs, with staff and resources being expanded beyond their intended allocation. By recognizing the capabilities of intelligent supportive robotics, healthcare providers have an opportunity to augment ED capabilities through the use of strong leaders and appropriate implementation strategies. Allocating further research towards the use of supportive robotics impacting workplace practices within EDs will assist healthcare practitioners in recognizing the benefits of incorporating such technologies, allowing them to take the first step to counteract the beginnings of this crisis using supportive robotics.

\section{REFERENCES}

1. Cook AM, Polgar JM. Assistive technologies: principles and practice. 4th ed. E-book. Elsevier Health Sciences; 2014 Nov 14. 488 p.

2. Merriam-Webster. Robot [Internet]. 2018 [cited 2018 July 30]. Available from: https://www.merriam-webster.com/dictionary/robot.

3. Gurbutt R, Roberts J. Is nursing decision making adequately supported in informatics solutions today? Br J Healthc Comput Inform Manag. 2006 Mar; 23(2):16.

4. Mihailidis A, Krones L, Boger J. Assistive computing devices: a pilot study to explore nurses' preferences and needs. Comput Inform Nurs. 2006 Nov 1;24(6):328-36.

5. Bettinelli M, Lei Y, Beane $\mathrm{M}$, et al. Does robotic telerounding enhance nurse-physician collaboration satisfaction about care decisions?. Telemed E Health. 2015 Aug 1;21(8):637-43. https://doi.org/10.1089/ tmj.2014.0162

6. Liao PH, Hsu PT, Chu W, et al. Applying artificial intelligence technology to support decision-making in nursing: A case study in Taiwan. Health Inform J. 2015 Jun;21(2):137-48. https://doi. org/10.1177/1460458213509806

7. Wilkes DM, Franklin S, Erdemir E, et al. Heterogeneous artificial agents for Triage nurse assistance. In Humanoid Robots (Humanoids), 2010 10th IEEE-RAS International Conference on 2010 Dec 6 (pp. 130137). Nashville, TN. IEEE 2011. p. 130-7.

8. Canadian Institute for Health Information. Emergency department wait times in Canada continuing to rise [Internet]. 2018 [cited 2018 July 25]. Available from https://www.cihi.ca/en/emergencydepartment-wait-times-in-canada-continuing-to-rise.

9. Canadian Institute for Health Information. Report. Regulated Nurses, 2016. Ottawa, ON: CIHI; 2017 [cited 2018 July 20]. 32 p. Available from https://www.cihi.ca/sites/default/files/document/regulated-nurses2016-report-en-web.pdf.

10. Health Quality Ontario. Report. Under Pressure: Emergency department performance in Ontario. Toronto: Queen's Printer for Ontario; 2016 [cited 2018 July 25]. 46 p. Available from: http://www. hqontario.ca/portals/0/Documents/system-performance/underpressure-report-en.pdf.

11. Transaction Data Systems, Inc. Kirby Lester [Internet]. 2018 [cited 2018 July 30]. Available from https://www.rx30.com/interfacesmodules/robotics-interfaces/kirby-lester/.
12. Kobayashi LC, Wardle J, von Wagner C. Internet use, social engagement and health literacy decline during ageing in a longitudinal cohort of older English adults. J Epidemiol Community Health. 2014 Nov;8. http://dx.doi.org/10.1136/jech-2014-204733

13. Tennant B, Stellefson M, Dodd V, et al. eHealth literacy and Web 2.0 health information seeking behaviors among baby boomers and older adults. J Med Internet Res. 2015 Mar;17(3). https://doi.org/10.2196/ jmir.3992

14. McCartney M. Margaret McCartney: Nurses must be allowed to exercise professional judgment. BMJ. 2017 Mar 28;356:1548.https:// doi-org.proxyl.lib.uwo.ca/10.1136/bmj.j1548

15. Årstad I, Aven T. Managing major accident risk: concerns about complacency and complexity in practice. Saf Sci. 2017 Jan 1;91:114-21. https://doi.org/10.1016/j.ssci.2016.08.004 